\title{
Volatile Compounds in Fruit Peels as Novel Biomarkers for the Identification of Four Citrus Species
}

\author{
Haipeng Zhang, Huan Wen, Jiajing Chen, Zhaoxin Peng, Meiyan Shi, Mengjun Chen, Ziyu Yuan, \\ Yuan Liu, Hongyan Zhang and Juan $\mathrm{Xu} * \mathbb{D}$ \\ Key Laboratory of Horticultural Plant Biology (Ministry of Education), College of Horticulture and Forestry, \\ Huazhong Agricultural University, Wuhan 430070, China; haipengzhang@webmail.hzau.edu.cn (H.Z.); \\ ndwenwen@163.com (H.W.); jiajingchen@webmail.hzau.edu.cn (J.C.); pengzhaoxin@webmail.hzau.edu.cn (Z.P.); \\ shimeiyan@webmail.hzau.edu.cn (M.S.); c18186100353@163.com (M.C.); yuanziyu@webmail.hzau.edu.cn (Z.Y.); \\ luiyuer@webmail.hzau.edu.cn (Y.L.); zhanghy@mail.hzau.edu.cn (H.Z.) \\ * Correspondence: xujuan@mail.hzau.edu.cn; Tel.: +86-27-87286965
}

Academic Editors: José Sousa Câmara, Rosa Perestrelo and Jorge Pereira

Received: 20 November 2019; Accepted: 10 December 2019; Published: 12 December 2019

check for updates

\begin{abstract}
The aroma quality of citrus fruit is determined by volatile compounds, which bring about different notes to allow discrimination among different citrus species. However, the volatiles with various aromatic traits specific to different citrus species have not been identified. In this study, volatile profiles in the fruit peels of four citrus species collected from our previous studies were subjected to various analyses to mine volatile biomarkers. Principal component analysis results indicated that different citrus species could almost completely be separated. Thirty volatiles were identified as potential biomarkers in discriminating loose-skin mandarin, sweet orange, pomelo, and lemon, while 17 were identified as effective biomarkers in discriminating clementine mandarins from the other loose-skin mandarins and sweet oranges. Finally, 30 citrus germplasms were used to verify the classification based on $\beta$-elemene, valencene, nootkatone, and limettin as biomarkers. The accuracy values were $90.0 \%, 96.7 \%, 96.7 \%$, and $100 \%$, respectively. This research may provide a novel and effective alternative approach to identifying citrus genetic resources.
\end{abstract}

Keywords: citrus; volatiles; biomarkers

\section{Introduction}

Citrus is one of the most important fruit tree genera in the world; Citrus acreage and production are top-ranked globally [1]. Citrus fruit are well accepted by consumers because of their nutritional and health-promoting properties. In citrus fruit, bioactive compounds, including vitamin C, soluble sugars, organic acids, amino acids, flavonoids, carotenoids, and volatile compounds, contribute to the global fruit quality while also being beneficial to human health [1-8]. Other than their contribution to the odor notes, volatile compounds play important roles in the interactions between plants and their environment [9] via attracting pollinators and defending against pathogens and herbivores [10-12].

Citrus fruit peels are rich in volatile compounds, mainly including terpenoids, aldehydes, alcohols, acids, and esters [8]. Terpenoids are the most abundant volatiles, accounting for more than $90 \%$ in most citrus germplasms [8]. Both the composition and contents of volatile compounds are important in affecting the fruit odor and sensory properties. For instance, the contents of 16 volatile compounds were significantly changed in the fruit of Niurouhong (Citrus reticulata Blanco) in comparison with its wild type, Zhuhongju, resulting in significant changes in the aroma traits [13]. The contents of cisand trans-linalool oxides in Huanong Red pomelo fruit increased after being pollinated with Citrus 
mangshanensis, which consequently obtained the characteristic aroma of its pollen parent [14]. By using partial least squares discriminant analysis (PLS-DA), 15 compounds were selected as metabolite markers to distinguish between Chenpi (C. reticulata Blanco) and Guangchenpi (C. reticulata 'Chachi') fruit peel [15].

As one of the original centers of Citrus, China has many citrus germplasm resources, mainly including sweet orange (C. sinensis), loose-skin mandarin (C. reticulata), lemon (C. limon), and pomelo (C. grandis) [1,16]. Furthermore, some hybrid citrus germplasms, such as clementine mandarin (C. clementina Hort. ex Tanaka), which may be a hybrid of loose-skin mandarin and sweet orange [17,18], are also widely cultivated. It is known that the fruit of each citrus species has a recognizable aroma trait. For example, the aromas of fruit are quite similar in different sweet orange germplasms but different from loose-skin mandarin, pomelo, and lemon accessions. The aroma of clementine mandarin is different from both parents; however, to our knowledge, the specific volatile compounds accounting for the differences have not been identified.

Previous reports have focused on the identification of key characteristic aroma compounds that contribute to the specific flavor [19-23]. In these reports, valencene was taken as a characteristic aromatic compound in sweet orange fruit [23], cis- and trans-linalool oxides and $\beta$-myrcene were the main characteristic aromatic compounds in C. mangshanensis fruit [21], $\beta$-pinene, $\gamma$-terpinene, linalyl acetate, and linalool contributed to the characteristic aroma of lemon fruit [22], $\beta$-citronellal, nerol acetate, and geranyl acetate were mainly responsible for the characteristic aroma of lime fruit [20,22], and linalool and its derivatives can largely reconstruct the sweet and fragrant aroma of the Miyamoto satsuma mandarin (C. reticulata) [19]. All of these characteristic volatiles can serve as biomarkers to distinguish the specific citrus germplasm. However, to our knowledge, these studies only focused on limited citrus germplasms, and the characteristic aromatic compounds may not be species specific.

Hierarchical clustering analysis (HCA), principal component analysis (PCA), and PLS-DA have been effectively used to analyze the relationship between different citrus species based on metabolomics data $[8,24,25]$. PLS-DA has been used to determine the differentially accumulated compounds in different species, where the compounds with a high variable importance in projection (VIP) value were selected as the potential specific biomarkers [15].

In the study, large-scaled volatile compound data from 66 citrus germplasms representing four citrus species were used to mine the potential species-specific volatile biomarkers. PCA and PLS-DA were applied in choosing the important volatiles that contribute to the specific volatile profile of different citrus species, and a total of 30 compounds were selected as potential biomarkers. Thirty citrus germplasms were used for classification, and a high level of accuracy was found, based on four volatile compounds with high VIP values.

\section{Results}

\subsection{Volatile Compounds in Fruit Peels}

In total, 89 volatile compounds (see Supplementary Materials) from four citrus species were used for analysis. The Venn plot indicated that all of them were detected in at least two citrus species, and 59 volatile compounds were commonly detected in four species (Figure 1A), indicating that the volatile compounds identified were similar in different citrus species. 
A

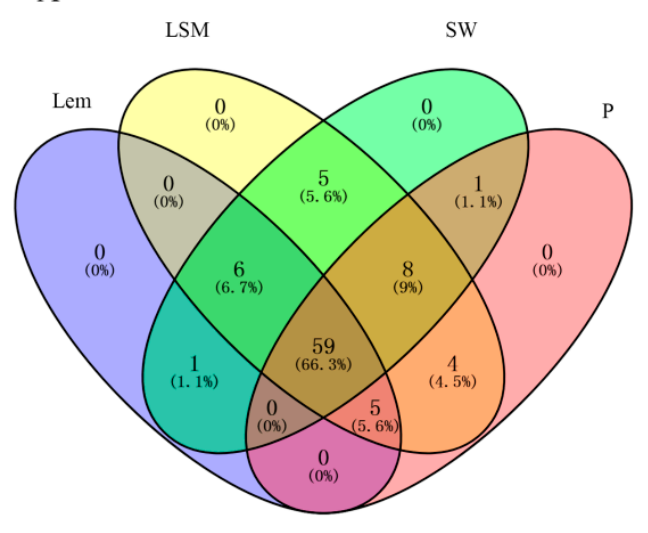

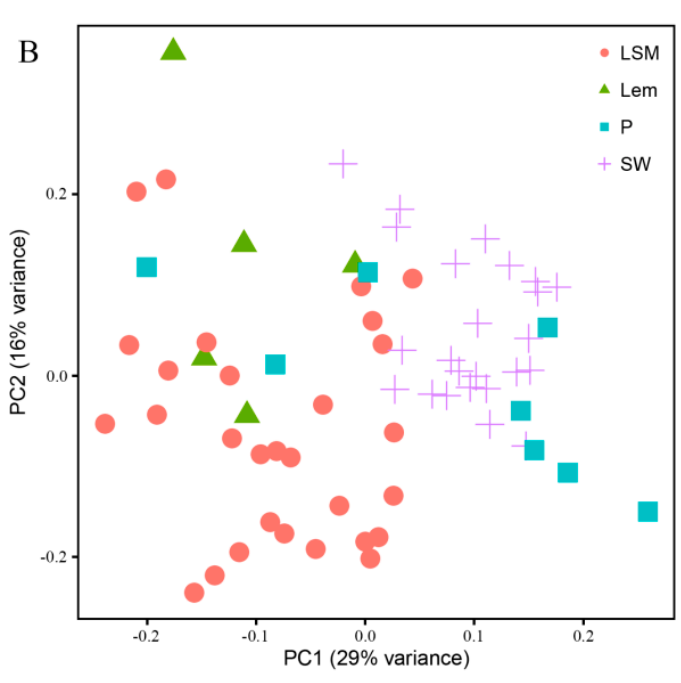

Figure 1. Volatile compounds in four citrus species. (A) Venn plot of the volatile compounds in four citrus species and (B) principal component analysis (PCA) results of the volatile profiles in four citrus species. SW: sweet orange; P: pomelo; LSM: loose-skin mandarin; Lem: lemon.

The PCA results showed that the first principal component explained $29 \%$ of the varianceloose-skin mandarin (LSM) and lemon (Lem) were clearly separated from pomelo (P) and sweet orange (SW) on the PC1 axis. The second principal component explained $16 \%$ of the variance-SW was clearly separated from P, while LSM was separated from Lem on the PC2 axis (Figure 1B). Although the first two principal components explained only approximately $45 \%$ of the variance, four citrus species (LSM, SW, P, and Lem) used in the study were clearly distinguished from each other. The results indicated that different citrus species were indeed species specific with regard to their volatile profiles.

\subsection{Accumulation Pattern of Volatile Compounds is Citrus Species Dependent}

To identify the potential volatile biomarkers that discriminate different citrus species, the volatile profiles of LSM, SW, P, and Lem were applied. The results indicated that almost all of them were grouped into a citrus species and separated from the other citrus species by a PLS-DA loading plot (Figure 2). In total, 30 potential volatile biomarkers were found that might be used to distinguish different citrus species (Table 1), and 17 potential volatile biomarkers contributed to the difference between clementine mandarin and its parents (LSM and SW) (see Supplementary Materials). 

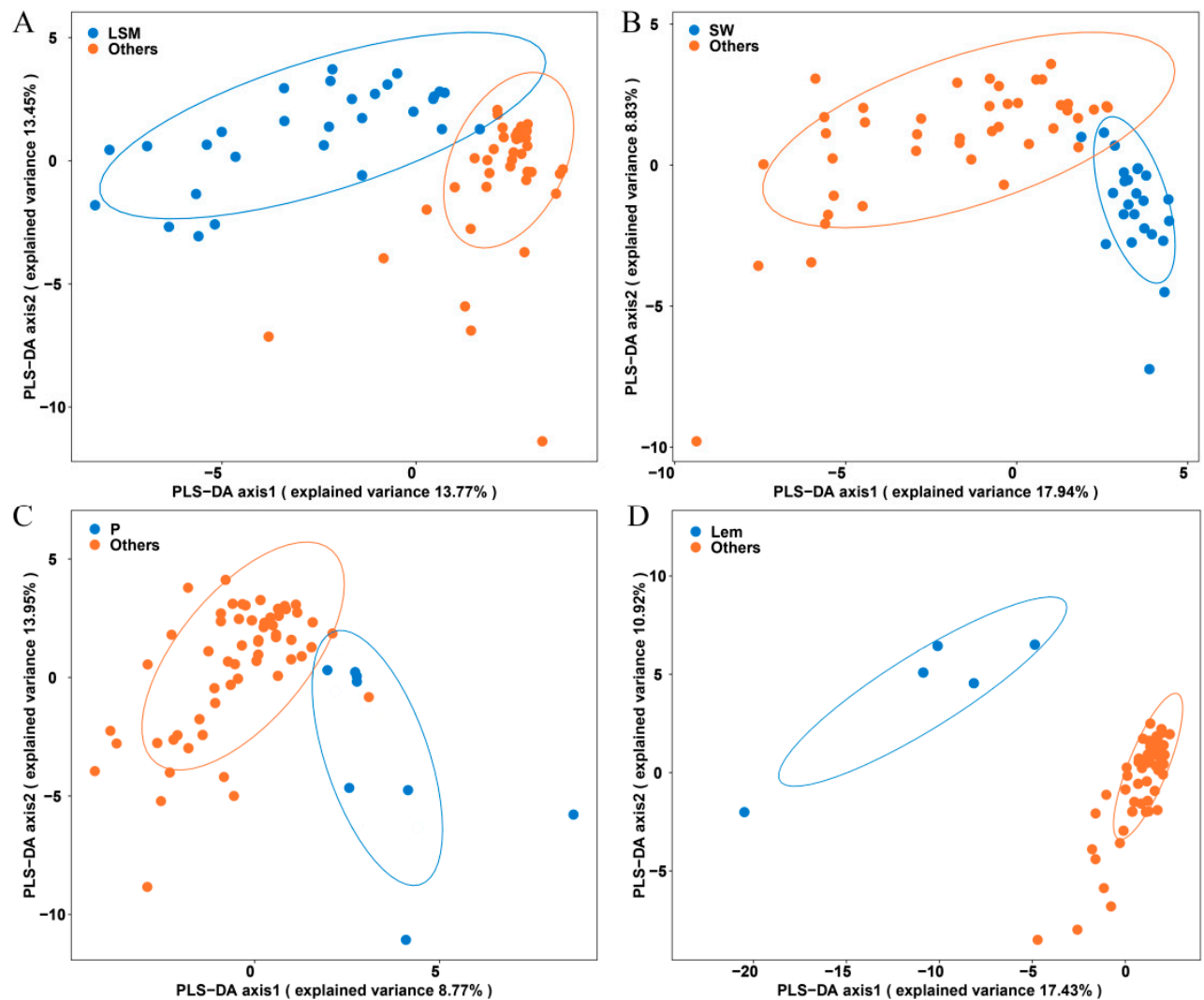

Figure 2. Partial least squares discriminant analysis (PLS-DA) score plots of four citrus species. (A) The results of the PLS-DA clearly distinguished the LSM and the other three citrus species using volatile profiles. (B-D) The results of the PLS-DA clearly distinguished the SW/P/Lem and the other three citrus species using volatile profiles, respectively. LSM: loose-skin mandarin; SW: sweet orange; P: pomelo; Lem: lemon; O: other citrus species.

Table 1. Potential biomarkers selected in four citrus species.

\begin{tabular}{|c|c|c|c|c|c|c|c|}
\hline ID & Compounds & VIP & P-Value & ID & Compounds & VIP & $P$-Value \\
\hline \multicolumn{4}{|c|}{ Loose-skin mandarin (LSM) } & \multicolumn{4}{|c|}{ Lemon (Lem) } \\
\hline $\mathrm{C} 44$ & $\beta$-elemene & 1.98 & $3.85 \times 10^{-10}$ & $\mathrm{C} 89$ & limettin & 2.51 & $8.00 \times 10^{-16}$ \\
\hline $\mathrm{C} 38$ & valencene & 1.75 & $4.67 \times 10^{-6}$ & $\mathrm{C} 22$ & citronellal & 2.40 & $2.91 \times 10^{-4}$ \\
\hline $\mathrm{C} 36$ & $(Z)-\beta$-farnesene & 1.63 & $7.15 \times 10^{-6}$ & C47 & trans- $\alpha$-bergamotene & 2.30 & $1.26 \times 10^{-13}$ \\
\hline C61 & caryophyllene oxide & 1.63 & $2.76 \times 10^{-4}$ & $\mathrm{C} 54$ & $\beta$-bisabolene & 2.22 & $1.26 \times 10^{-13}$ \\
\hline C56 & germacrene B & 1.60 & $5.95 \times 10^{-7}$ & $\mathrm{C} 45$ & cis- $\alpha$-bergamotene & 1.96 & $5.52 \times 10^{-9}$ \\
\hline $\mathrm{C} 73$ & 3-hexenal & 1.56 & $3.69 \times 10^{-2}$ & $\mathrm{C} 3$ & $\beta$-pinene & 1.95 & $1.56 \times 10^{-3}$ \\
\hline $\mathrm{C} 46$ & $\gamma$-elemene & 1.54 & $9.47 \times 10^{-5}$ & C35 & caryophyllene & 1.89 & $9.80 \times 10^{-4}$ \\
\hline $\mathrm{C} 37$ & $\alpha$-caryophyllene & 1.51 & $7.68 \times 10^{-10}$ & $\mathrm{C} 13$ & camphene & 1.81 & $2.00 \times 10^{-3}$ \\
\hline $\mathrm{C} 40$ & $\delta$-elemene & 1.50 & $1.86 \times 10^{-7}$ & C41 & $\alpha$-cubebene & 1.71 & $1.15 \times 10^{-4}$ \\
\hline \multirow[t]{2}{*}{$\mathrm{C} 10$} & $\gamma$-terpinene & 1.50 & $8.75 \times 10^{-4}$ & $\mathrm{C} 18$ & $\alpha$-terpineol & 1.57 & $1.03 \times 10^{-3}$ \\
\hline & \multicolumn{3}{|c|}{ Sweet orange (SW) } & $\mathrm{C} 36$ & $(Z)$ - $\beta$-farnesene & 1.56 & $4.24 \times 10^{-3}$ \\
\hline $\mathrm{C} 38$ & valencene & 2.54 & $6.19 \times 10^{-9}$ & \multicolumn{4}{|c|}{ Pomelo (P) } \\
\hline C61 & caryophyllene oxide & 2.44 & $2.90 \times 10^{-11}$ & $\mathrm{C} 88$ & nootkanone & 3.07 & $1.13 \times 10^{-5}$ \\
\hline $\mathrm{C} 10$ & $\gamma$-terpinene & 1.61 & $2.16 \times 10^{-8}$ & $\mathrm{C} 73$ & 3-hexenal & 2.01 & $1.51 \times 10^{-3}$ \\
\hline C5 & $\alpha$-phellandrene & 1.56 & $5.68 \times 10^{-5}$ & C30 & $\begin{array}{l}\text { trans-limonene } \\
\text { oxide }\end{array}$ & 1.87 & $2.70 \times 10^{-3}$ \\
\hline $\mathrm{C} 12$ & $\alpha$-thujene & 1.53 & $2.61 \times 10^{-8}$ & $\mathrm{C} 43$ & $\beta$-cubebene & 1.77 & $2.07 \times 10^{-3}$ \\
\hline $\mathrm{C} 30$ & trans-limonene oxide & 1.53 & $9.49 \times 10^{-4}$ & C58 & elemol & 1.77 & $5.16 \times 10^{-4}$ \\
\hline C49 & germacrene D & 1.52 & $1.38 \times 10^{-7}$ & $\mathrm{C} 42$ & copaene & 1.61 & $4.16 \times 10^{-3}$ \\
\hline C6 & $\alpha$-terpinene & 1.50 & $2.05 \times 10^{-7}$ & & & & \\
\hline
\end{tabular}

\subsubsection{Discrimination of LSM from the Other Three Citrus Species}

To discriminate LSM from the other citrus species and find the potential markers responsible for such classification, PLS-DA was employed. The PLS-DA loading plot showed that the volatile 
profiles of LSM and the other citrus species could be separated (Figure 2A), indicating that the volatile compositions of LSM and the other citrus species were quite different. The volatile markers were selected based on the VIP value (Table 1).

Consequently, 10 volatile compounds with a VIP value greater than 1.5 were selected as biomarker compounds that were responsible for the discrimination of LSM from the other citrus species. As shown in Figure 3A, the contents of $\beta$-elemene, germacrene B, 3-hexenal, $\gamma$-elemene, $\alpha$-caryophyllene, $\delta$-elemene, and $\gamma$-terpinene in LSM were significantly higher than those in the other citrus species, while the levels of valencene, (Z)- $\beta$-farnesene, and caryophyllene oxide in LSM were significantly lower than those in the other citrus species. These compounds might serve as potential biomarkers for the discrimination of LSM from the other citrus species.
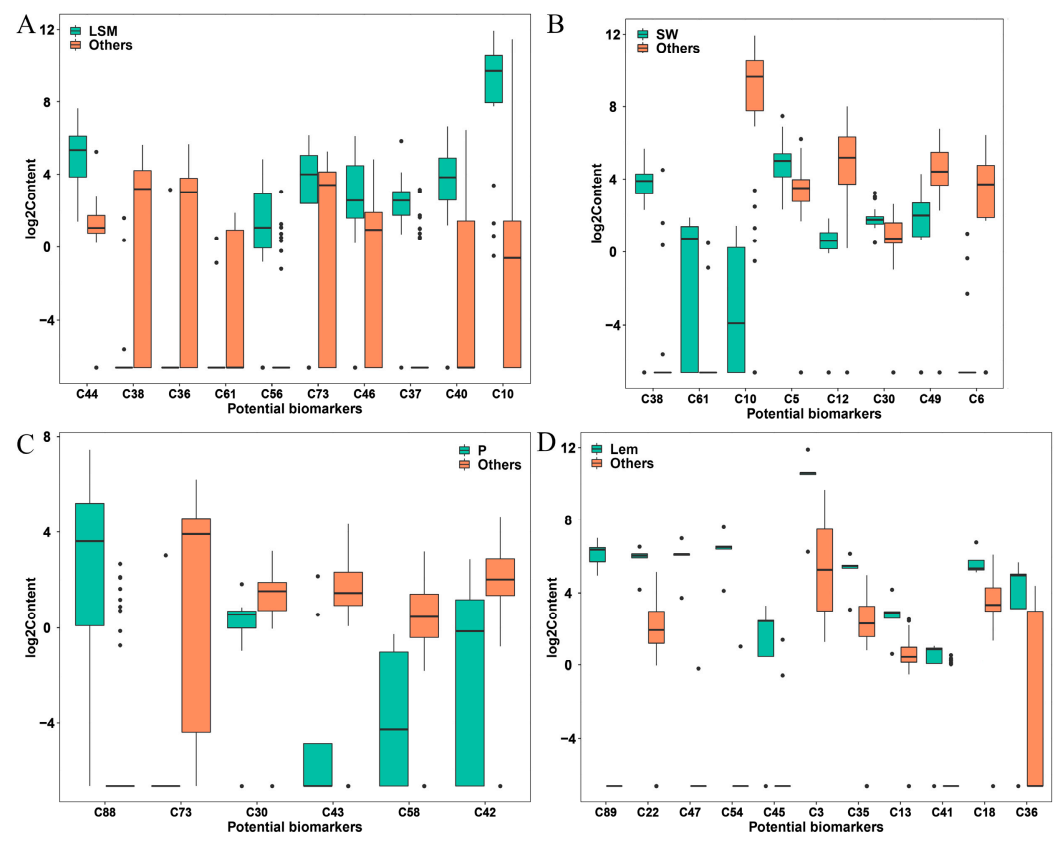

Figure 3. Boxplots showing the contents of biomarkers in LSM (A), SW (B), P (C), and Lem (D) and the other citrus germplasms. The biomarkers are listed in Table 1. LSM: loose-skin mandarin; SW: sweet orange; P: pomelo; Lem: lemon.

\subsubsection{Discrimination of Sweet Orange from the Other Three Citrus Species}

To find the potential biomarkers that contribute to the differences between SW and the other citrus species, the volatile profiles of 24 sweet oranges and the other 42 citrus germplasms were analyzed, and eight volatile compounds were selected as candidate biomarkers by PLS-DA (Figure 2B). The valencene and caryophyllene oxide were the most important compounds (VIP value $>2$ ) (Table 1). A comparison between the contents of the biomarkers in SW and the other citrus species found that the levels of valencene, caryophyllene oxide, $\alpha$-phellandrene, and trans-limonene oxide were high in SW, while the levels of $\gamma$-terpinene, $\alpha$-thujene, germacrene $\mathrm{D}$, and $\alpha$-terpinene were low (Figure 3B).

\subsubsection{Discrimination of Pomelo from the Other Three Citrus Species}

To investigate the difference between pomelo and the other citrus species, nine biomarker compounds were selected by PLS-DA (Figure 2C). Meanwhile, the significant differences were estimated in pomelo and the other citrus species, and six volatile compounds were identified by combining the VIP scores and P-values. Nootkatone and 3-hexenal were the most important compounds (VIP value $>2$ ) (Table 1). Among the six biomarker compounds, the content of nootkatone in pomelos was significantly higher than that in the other citrus species, while the levels of 3-hexenal, trans-limonene oxide, $\beta$-cubebene, elemol, and octyl ester were low in pomelos (Figure $3 \mathrm{C}$ ). 


\subsubsection{Discrimination of Lemon from the Other Three Citrus Species}

Eleven volatile compounds served as biomarkers to distinguish five lemons from the other 61 citrus germplasms (Figure 2D). Limettin, citronellal, trans- $\alpha$-bergamotene, and $\beta$-bisabolene were the most important compounds (VIP value $>2$ ) (Table 1). The contents of 11 biomarker compounds (limettin, citronellal, trans- $\alpha$-bergamotene, $\beta$-bisabolene, cis- $\alpha$-bergamotene, $\beta$-pinene, caryophyllene, camphene, $\alpha$-cubebene, $\alpha$-terpineol, and (Z)- $\beta$-farnesene) in lemons were significantly higher than those in the other citrus species (Figure 3D).

\subsubsection{Discrimination of Clementine Mandarin from LSM and SW}

Clementine mandarin is a hybrid cultivar from LSM and SW [16], with a specific aroma different from LSM and SW. To identify the specific compounds that contribute to the difference, 16 clementine mandarins, 29 LSMs, and 24 SWs were used, and 16 marker compounds were selected by PLS-DA (Figure 4; see Supplementary Materials). Dodecanal, decanal, (Z)- $\beta$-farnesene, $\alpha$-sinensal, ylangene, $\alpha$-muurolene, and $\alpha$-terpineol acetate were the important compounds (VIP value $>2$ ). It was found that the levels of all of these 16 markers were high in clementine mandarins (see Supplementary Materials).

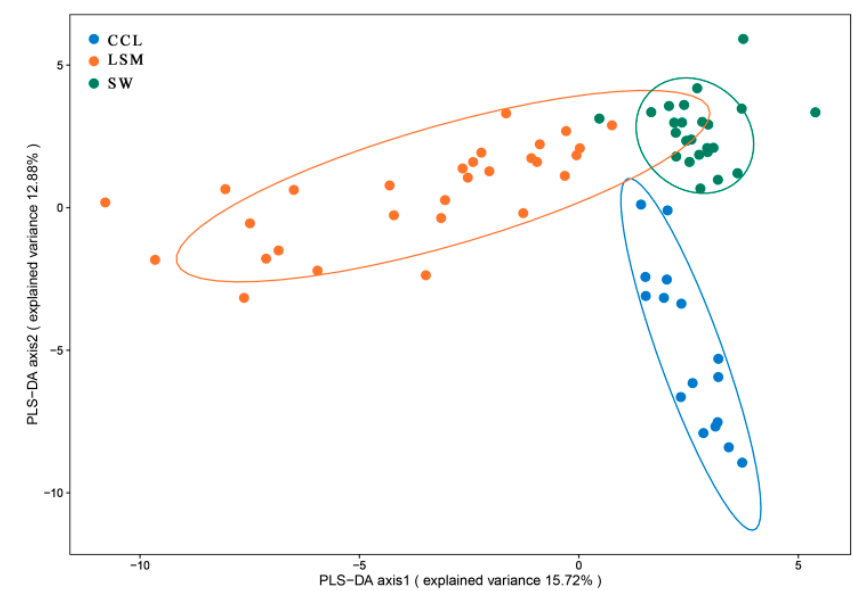

Figure 4. PLS-DA score plots of clementine mandarin, LSM, and SW. LSM: loose-skin mandarin; SW: sweet orange; CCL: clementine mandarin.

\subsection{Four Biomarkers for the Identification of Four Citrus Species}

Overall, $\beta$-elemene, valencene, nootkatone, and limettin were the most important compounds, and their contents were significantly different in LSM, SW, P, and Lem (Figure 5). To further verify the accuracy of the four biomarkers in the identification of four citrus species, 30 citrus germplasms collected in 2017 were used in the study. $\beta$-elemene was used as a marker to classify the 30 citrus germplasms into two groups-LSM or not LSM. The results showed that 27 citrus germplasms were correct, while the other three (Suhong tangerine, Hamlin sweet orange, and Kesai lime) were incorrect. The accuracy values were $90.0 \%, 96.7 \%, 96.7 \%$, and $100 \%$, based on $\beta$-elemene, valencene, nootkatone, and limettin as markers, respectively (Table 2). 


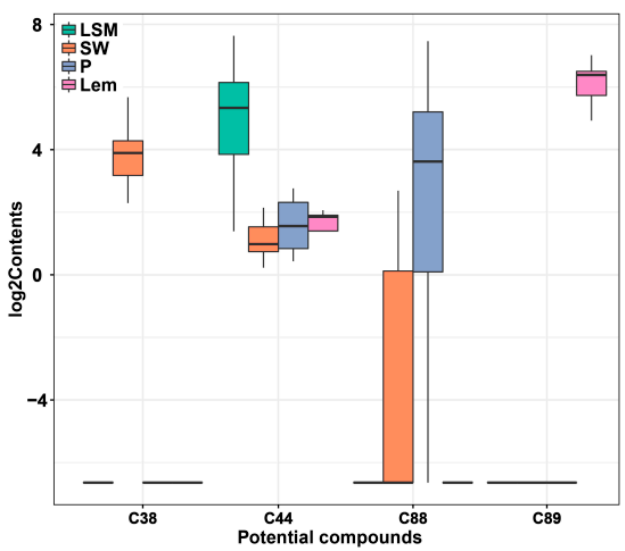

Figure 5. Boxplot showing the contents of four high variable importance in projection (VIP) value biomarkers in LSM, SW, P, and Lem. LSM: loose-skin mandarin; SW: sweet orange; P: pomelo; Lem: lemon. C38: valencene; C44: $\beta$-elemene; C88: nootkatone; C89: limettin.

Table 2. Four biomarkers for the identification of four citrus species.

\begin{tabular}{|c|c|c|c|c|c|}
\hline Citrus Germplasms & Actual & $\beta$-Elemene ${ }^{a}$ & Valencene $^{b}$ & Nootkanone $^{c}$ & Limettin $^{d}$ \\
\hline Fu tangerine & LSM & Yes $(Y)$ & $\mathrm{N}$ & $\mathrm{N}$ & $\mathrm{N}$ \\
\hline Huapi tangerine & LSM & $\mathrm{Y}$ & $\mathrm{N}$ & $\mathrm{N}$ & $\mathrm{N}$ \\
\hline Zhuhong tangerine & LSM & Y & $\mathrm{N}$ & $\mathrm{N}$ & $\mathrm{N}$ \\
\hline Nian tangerine & LSM & Y & $\mathrm{N}$ & $\mathrm{N}$ & $\mathrm{N}$ \\
\hline Shatang tangerine & LSM & $\mathrm{Y}$ & $\mathrm{N}$ & $\mathrm{N}$ & $\mathrm{N}$ \\
\hline Suhong tangerine & LSM & No $(\mathrm{N})$ & $\mathrm{N}$ & $\mathrm{N}$ & $\mathrm{N}$ \\
\hline Nanfengmi tangerine & LSM & $\mathrm{Y}$ & $\mathrm{N}$ & $\mathrm{N}$ & $\mathrm{N}$ \\
\hline Huanongbendizao tangerine & LSM & Y & $\mathrm{N}$ & $\mathrm{N}$ & $\mathrm{N}$ \\
\hline Tu tangerine & LSM & $\mathrm{Y}$ & $\mathrm{N}$ & $\mathrm{N}$ & $\mathrm{N}$ \\
\hline Red tangerine & LSM & $\mathrm{Y}$ & $\mathrm{N}$ & $\mathrm{N}$ & $\mathrm{N}$ \\
\hline Anliu sweet orange & SW & $\mathrm{N}$ & $\mathrm{Y}$ & $\mathrm{N}$ & $\mathrm{N}$ \\
\hline Valencia sweet orange & SW & $\mathrm{N}$ & $\mathrm{Y}$ & $\mathrm{N}$ & $\mathrm{N}$ \\
\hline Hamlin sweet orange & SW & $\mathrm{Y}$ & $\mathrm{Y}$ & $\mathrm{N}$ & $\mathrm{N}$ \\
\hline Hong anliu sweet orange & SW & $\mathrm{N}$ & $\mathrm{Y}$ & $\mathrm{N}$ & $\mathrm{N}$ \\
\hline Cara cara navel orange & SW & $\mathrm{N}$ & $\mathrm{Y}$ & $\mathrm{N}$ & $\mathrm{N}$ \\
\hline Jincheng sweet orange & SW & $\mathrm{N}$ & $\mathrm{Y}$ & $\mathrm{N}$ & $\mathrm{N}$ \\
\hline Washington navel orange & SW & $\mathrm{N}$ & $\mathrm{Y}$ & $\mathrm{N}$ & $\mathrm{N}$ \\
\hline Seika navel orange & SW & $\mathrm{N}$ & $\mathrm{Y}$ & $\mathrm{N}$ & $\mathrm{N}$ \\
\hline Newhall navel orange & SW & $\mathrm{N}$ & $\mathrm{Y}$ & $\mathrm{N}$ & $\mathrm{N}$ \\
\hline Feicui pomelo & $\mathrm{P}$ & $\mathrm{N}$ & $\mathrm{N}$ & $\mathrm{Y}$ & $\mathrm{N}$ \\
\hline Kaopan pomelo & $\mathrm{P}$ & $\mathrm{N}$ & $\mathrm{N}$ & $\mathrm{Y}$ & $\mathrm{N}$ \\
\hline Huanong red pomelo & $\mathrm{P}$ & $\mathrm{N}$ & $\mathrm{N}$ & $\mathrm{Y}$ & $\mathrm{N}$ \\
\hline Liangping pomelo & $\mathrm{P}$ & $\mathrm{N}$ & $\mathrm{N}$ & $\mathrm{Y}$ & $\mathrm{N}$ \\
\hline Wanbai pomelo & $\mathrm{P}$ & $\mathrm{N}$ & $\mathrm{N}$ & Y & $\mathrm{N}$ \\
\hline Low-acid pomelo & $\mathrm{P}$ & $\mathrm{N}$ & $\mathrm{N}$ & $\mathrm{Y}$ & $\mathrm{N}$ \\
\hline Finger citron & Lem & $\mathrm{N}$ & $\mathrm{N}$ & $\mathrm{N}$ & $\mathrm{Y}$ \\
\hline Tahiti lime & Lem & $\mathrm{N}$ & $\mathrm{N}$ & $\mathrm{Y}$ & $\mathrm{Y}$ \\
\hline Kesai lime & Lem & $\mathrm{Y}$ & Y & $\mathrm{N}$ & $\mathrm{Y}$ \\
\hline Verna lemon & Lem & $\mathrm{N}$ & $\mathrm{N}$ & $\mathrm{N}$ & $\mathrm{Y}$ \\
\hline Eureka lemon & Lem & $\mathrm{N}$ & $\mathrm{N}$ & $\mathrm{N}$ & Y \\
\hline Accuracy & & $90.0 \%$ & $96.7 \%$ & $96.7 \%$ & $100.00 \%$ \\
\hline
\end{tabular}

${ }^{a}$ The citrus germplasm was classified as loose-skin mandarin (LSM) or not, using $\beta$-elemene as a marker; ${ }^{\mathrm{b}}$ the citrus germplasm was classified as sweet orange (SW) or not, with valencene as a marker; ${ }^{c}$ the citrus germplasm was classified as pomelo $(\mathrm{P})$ or not, using nootkanone as a marker; and ${ }^{\mathrm{d}}$ the citrus germplasm was classified as lemon (Lem) or not, using limettin as a marker.

\subsection{Discrimination of Wild and Cultivar Germplasms}

Twenty compounds were selected as biomarkers that contributed to the discrimination of wild and cultivar germplasms by PLS-DA. Germacrene B, $\gamma$-elemene, and trans-nerolidol were the important 
compounds with VIP values $>2$ (see Supplementary Materials). The contents of 19 marker compounds were high, while only one had a low level in wild germplasms (see Supplementary Materials).

\section{Discussion}

\subsection{Biomarkers for Discriminating between Different Citrus Germplasms}

Lots of germplasms in Citrus, including mainly LSM, sweet orange, pomelo, lemon, and various hybrid germplasms, were used in the study [1]. For example, the fruit shape and size of clementine mandarin were similar to LSM but had a different aroma. The contents of 14 biomarker compounds in clementine mandarin were significantly higher than those in LSM and sweet orange (see Supplementary Materials). The results indicated that these 14 compounds may contribute to the specific aroma of clementine mandarins and can also be used as biomarkers to distinguish clementine mandarin from LSM and sweet orange. Due to more and more hybrid germplasms having been released from citrus breeding programs, it is hard to distinguish them from each other just based on fruit shape and size. The volatile biomarkers may thus be a good method for discrimination between them. In total, 30 volatile biomarkers were found to distinguish between different citrus species (Table 1).

Furthermore, four compounds with the highest VIP values were selected as biomarkers to discriminate between 30 citrus germplasms. The accuracy of the identification results was very high, with only three, one, one, and zero being incorrect, based on $\beta$-elemene, valencene, nootkatone, and limettin, respectively (Table 2). Therefore, with the use of volatile compounds as biomarkers for the identification of citrus germplasms shown to be reliable and with the method being cheaper, simpler, faster, and easier, using volatile compounds might serve as an alternative or primary method to the molecular methods (simple sequence repeats and single-nucleotide polymorphisms).

\subsection{Biomarkers May Be Responsible for the Citrus Species-Specific Odor Notes}

There were abundant volatile compounds in citrus fruit. Lots of reports have mainly focused on the determination of volatile compounds and the comparison of the number and content of them in a few citrus germplasms [8,13,21,24-27]. As different citrus species may have a unique aroma, the profiles of volatiles have been used to study citrus chemotaxonomy [8,24,25]. In addition, some researchers have identified the characteristic aroma compounds in citrus germplasms with specific aroma traits, such as C. mangshanensis, sweet orange, lemon, and lime [19-23].

It has been reported that valencene is a characteristic aroma compound in sweet orange $[23,28]$. In the study, a high VIP value (2.54) of valencene was found by PLS-DA (Table 1, Figure 3B). Valencene was mainly accumulated in sweet orange, indicating that valencene may contribute to the characteristic aroma of sweet orange. In addition, the levels of some biomarker compounds were high or low in SW fruit (Table 1, Figure 3B), suggesting that these compounds may also contribute to its specific aroma. Furthermore, ten, eleven, and seven biomarker compounds were selected in LSM, lemon, and pomelo, respectively (Table 1). The different accumulation of these compounds in different citrus species may be responsible for the species-specific aroma.

Although some candidate compounds that might contribute to the specific aroma were selected by PLS-DA (Table 1), the contribution of each compound was still unclear. To further determine the effects of these compounds, gas chromatography-olfactory (GC-O), aroma extraction dilution analysis (AEDA), and odor activity analysis were required [21]. The specific aroma of citrus fruit does not result from one specific volatile. For example, the specific balsamic and floral odor of $C$. mangshanensis fruit results from $d$-limonene as a background aroma, trans- and cis-linalool oxides, and $\beta$-myrcene [21].

\subsection{Protection and Utilization of Wild Citrus Germplasms}

In the citrus breeding process, the traits of fruit yield, maturity, and color are more likely to attract the attention of breeders. In flavor, the contents of sugar and acids are the most important, with the aroma trait having often been ignored in citrus breeding history. The levels of 19 of the 20 compounds 
in cultivars were lower than in wild germplasms. Similarly, the characteristic aroma compounds of C. mangshanensis (trans- and cis-linalool oxides) were detected in some wild citrus germplasms but not in cultivars [21]. Some of the compounds with decreased levels may contribute to the aroma odor in citrus fruit, such as linalool, trans-nerolidol, citronellal, and $\gamma$-terpinene. It was found that 18 of the 19 compounds were terpene compounds, and many terpene compounds play important roles in interactions with the environment, such as attracting pollinators and defending against pathogens and herbivores $[10,12,29]$. Therefore, the decreased levels of these volatile compounds might result from changes in the living environment. The wild citrus germplasms with good aroma traits may not only be used in citrus breeding but also provide raw materials for the extraction of essential oil.

\section{Materials and Methods}

\subsection{Materials}

The raw data of volatile compounds in the citrus peels were downloaded from our previous study [8]. At least five representative germplasms were selected from each citrus species, and as a result, volatile data from 29 loose-skin mandarins (LSMs), 24 sweet oranges (SWs), eight pomelos (Ps) and five lemons (Lems) (see Supplementary Materials) were used in the study. The volatile compounds that were detected in at least five germplasms were selected. Additionally, 16 clementine mandarins were used to analyze the difference between LSM and SW.

The fruits of 30 citrus germplasms were collected from the National Citrus Breeding Center (Wuhan, Hubei, China) and the Citrus Research Institute, Chinese Academy of Agricultural Sciences (Beibei, Chongqing, China), including 10 LSMs, nine sweet oranges, six pomelos, and five lemons. The fruit peels were separated and placed in liquid nitrogen and then stored at $-80^{\circ} \mathrm{C}$.

\subsection{Extraction and Determination of Volatiles}

According to the method of Zhang et al. [8], the volatile compounds were extracted by methyl tert-butyl ether (MTBE, HPLC grade) from $1 \mathrm{~g}$ of citrus peels. The TRACE GC Ultra GC coupled with a DSQ II mass spectrometer (Thermo Fisher Scientific, Waltham, MA, USA) with a TRACE TR-5 MS column ( $30 \mathrm{~m} \times 0.25 \mathrm{~mm} \times 0.25 \mu \mathrm{m}$; Thermo Scientific, Bellefonte, PA, USA) were used to obtain the profiles of the volatile compounds.

\subsection{Data Analysis}

A Venn plot was drawn using VENNY 2.1 [30]. Principal component analysis (PCA) by image GP (http://www.ehbio.com/ImageGP/index.php/Home/Index/index.html) was used to determine the volatile profiles of 66 citrus germplasms.

PLS-DA analysis was conducted using the R package mixOmics [31] and SIMCA-P software (Umetrics AB, Umea, Sweden). For example, to understand the difference of volatile compounds between LSM and the other citrus species, 29 LSMs and 37 other citrus germplasms were used to calculate the VIP value for each volatile compound by the PLS-DA method. A compound was selected when its VIP value was greater than 1.5. The specific compounds with high VIP values were used to distinguish between different citrus germplasms, and the difference was further verified using the volatile profiles with the $\mathrm{R}$ package ggplot2 and reshape2. R packages (nortest, stats, and pgirmess) were used for the ANOVA $(p<0.05)$.

To verify the accuracy of four biomarkers in discriminating between citrus species, a citrus germplasm was classified as LSM if its content of $\beta$-elemene was higher than $16 \mathrm{ng} / \mathrm{g}$ and as SW, P, or Lem when valencene, nootkatone, or limettin was detected.

\section{Conclusions}

The volatiles in the peels of 66 citrus germplasms from four citrus species were used for biomarker mining, and 30 potential biomarkers with different accumulation patterns in different citrus species 
were chosen using PLS-DA. The $\beta$-elemene, valencene, nootkatone, and limettin had the highest VIP values and were chosen as biomarkers for the identification of citrus species. An accuracy of $90.0 \%$, $96.7 \%, 96.7 \%$, and $100 \%$ in loose-skin mandarin, sweet orange, pomelo, and lemon was obtained, respectively. These biomarker compounds may be responsible for the specific aroma in different citrus species. This method is a novel and effective alternative approach to identifying citrus genetic resources with biomarkers.

Supplementary Materials: The following are available online: Table S1: Materials used in this study; Table S2: Volatile compounds used in the PLS-DA in this study; Table S3: Potential biomarkers selected in clementine mandarin and wild citrus germplasms; Figure S1: Boxplot showing the contents of biomarkers in clementine mandarin, LSM, and SW. The biomarkers are listed in Table S3; and Figure S2: Boxplot showing the contents of biomarkers in wild and cultivar germplasms. The biomarkers are listed in Table S3.

Author Contributions: Conceptualization, H.Z. (Hongyan Zhang) and J.X.; methodology, H.Z. (Hongyan Zhang); software, H.Z. (Haipeng Zhang); validation, H.Z. (Hongyan Zhang); formal analysis, Y.L.; investigation, H.W. and J.C.; resources, Z.P., M.S., M.C., and Z.Y.; data curation, H.Z. (Haipeng Zhang); writing-original draft preparation, H.Z. (Haipeng Zhang); writing—review and editing, J.X.; visualization, H.Z. (Haipeng Zhang); supervision, J.X.; project administration, J.X.; funding acquisition, J.X.

Funding: This research was funded by the National Key Research and Development Program (2018YFD1000200) and the National Natural Science Foundation of China (NSFC 31272122).

Acknowledgments: We thank Shiping Zhu of the Chinese Academy of Agricultural Sciences Citrus Research Institute for her help in the sample collection.

Conflicts of Interest: The authors declare no conflict of interest.

\section{References}

1. Xu, Q.; Chen, L.L.; Ruan, X.; Chen, D.; Zhu, A.; Chen, C.; Bertrand, D.; Jiao, W.B.; Hao, B.H.; Lyon, M.P. The draft genome of sweet orange (Citrus sinensis). Nat. Genet. 2013, 45, 59. [CrossRef] [PubMed]

2. Chen, J.; Zhang, H.; Pang, Y.; Cheng, Y.; Deng, X.; Xu, J. Comparative study of flavonoid production in lycopene-accumulated and blonde-flesh sweet oranges (Citrus sinensis) during fruit development. Food Chem. 2015, 184, 238-246. [CrossRef] [PubMed]

3. Huang, D.; Wang, X.; Tang, Z.; Yuan, Y.; Xu, Y.; He, J.; Jiang, X.; Peng, S.-A.; Li, L.; Butelli, E. Subfunctionalization of the Ruby2-Ruby1 gene cluster during the domestication of citrus. Nat. plants 2018, 4, 930. [CrossRef] [PubMed]

4. Liu, C.; Yan, F.; Gao, H.; He, M.; Wang, Z.; Cheng, Y.; Deng, X.; Xu, J. Features of citrus terpenoid production as revealed by carotenoid, limonoid and aroma profiles of two pummelos (Citrus maxima) with different flesh color. J. Sci. Food Agr. 2015, 95, 111-119. [CrossRef]

5. Tholl, D. Terpene synthases and the regulation, diversity and biological roles of terpene metabolism. Curr. Opin. Plant. Biol. 2006, 9, 297-304. [CrossRef]

6. Wang, S.; Tu, H.; Wan, J.; Chen, W.; Liu, X.; Luo, J.; Xu, J.; Zhang, H. Spatio-temporal distribution and natural variation of metabolites in citrus fruits. Food Chem. 2016, 199, 8-17. [CrossRef]

7. Xu, J.; Ma, L.; Jiang, D.; Zhu, S.; Yan, F.; Xie, Y.; Xie, Z.; Guo, W.; Deng, X. Content evaluation of 4 furanocoumarin monomers in various citrus germplasms. Food Chem. 2015, 187, 75-81. [CrossRef]

8. Zhang, H.; Xie, Y.; Liu, C.; Chen, S.; Hu, S.; Xie, Z.; Deng, X.; Xu, J. Comprehensive comparative analysis of volatile compounds in citrus fruits of different species. Food Chem. 2017, 230, 316-326. [CrossRef]

9. Baldwin, I.T. Plant volatiles. Curr. Biol. 2010, 20, 392-397. [CrossRef]

10. Ding, Y.; Huffaker, A.; Köllner, T.G.; Weckwerth, P.; Robert, C.A.M.; Spencer, J.L.; Lipka, A.E.; Schmelz, E.A. Selinene Volatiles Are Essential Precursors for Maize Defense Promoting Fungal Pathogen Resistance. Plant. Physiol. 2017, 175, 1455-1468. [CrossRef]

11. Dudareva, N.; Pichersky, E.; Gershenzon, J. Biochemistry of plant volatiles. Plant. Physiol. 2004, 135, 1893-1902. [CrossRef] [PubMed]

12. Rodriguez, A.; San Andres, V.; Cervera, M.; Redondo, A.; Alquezar, B.; Shimada, T.; Gadea, J.; Rodrigo, M.J.; Zacarias, L.; Palou, L.; et al. Terpene down-regulation in orange reveals the role of fruit aromas in mediating interactions with insect herbivores and pathogens. Plant. Physiol. 2011, 156, 793-802. [CrossRef] [PubMed] 
13. Li, W.; Liu, C.; He, M.; Li, J.; Cai, Y.; Ma, Y.; Xu, J. Largely different contents of terpenoids in beef red-flesh tangerine and its wild type. BMC Plant. Biol. 2017, 17, 36. [CrossRef] [PubMed]

14. Zhang, H.; Liu, C.; Yao, J.-L.; Deng, C.H.; Chen, S.; Chen, J.; Wang, Z.; Yu, Q.; Cheng, Y.; Xu, J. Citrus mangshanensis Pollen Confers a Xenia Effect on Linalool Oxide Accumulation in Pummelo Fruit by Enhancing the Expression of a Cytochrome P450 78A7 Gene CitLO1. J. Agr. Food Chem. 2019, 67, 9468-9476. [CrossRef]

15. Duan, L.; Guo, L.; Dou, L.-L.; Zhou, C.-L.; Xu, F.-G.; Zheng, G.-D.; Li, P.; Liu, E.-H. Discrimination of Citrus reticulata Blanco and Citrus reticulata 'Chachi' by gas chromatograph-mass spectrometry based metabolomics approach. Food Chem. 2016, 212, 123-127. [CrossRef] [PubMed]

16. Wu, G.A.; Terol, J.; Ibanez, V.; López-García, A.; Pérez-Román, E.; Borredá, C.; Domingo, C.; Tadeo, F.R.; Carbonell-Caballero, J.; Alonso, R. Genomics of the origin and evolution of Citrus. Nature 2018, 554, 311. [CrossRef] [PubMed]

17. Nicolosi, E.; Deng, Z.N.; Gentile, A.; Malfa, S.L.; Continella, G.; Tribulato, E. Citrus phylogeny and genetic origin of important species as investigated by molecular markers. Theor. Appl. Genet. 2000, 100, 1155-1166. [CrossRef]

18. Samaan, L.G. Studies on the origin of Clementine tangerine (Citrus reticulata Blanco). Euphytica 1982, 31, 167-173. [CrossRef]

19. Choi, H.S. Volatile constituents of satsuma mandarins growing in Korea. Flavour Frag. J. 2004, 19, 406-412. [CrossRef]

20. Lan-Phi, N.T.; Shimamura, T.; Ukeda, H.; Sawamura, M. Chemical and aroma profiles of yuzu (Citrus junos) peel oils of different cultivars. Food Chem. 2009, 115, 1042-1047. [CrossRef]

21. Liu, C.; Cheng, Y.; Zhang, H.; Deng, X.; Chen, F.; Xu, J. Volatile constituents of wild citrus Mangshanyegan (Citrus nobilis Lauriro) peel oil. J. Agr. Food Chem. 2012, 60, 2617-2628. [CrossRef] [PubMed]

22. Lota, M.-L.; de Rocca Serra, D.; Tomi, F.; Jacquemond, C.; Casanova, J. Volatile components of peel and leaf oils of lemon and lime species. J. Agr. Food Chem. 2002, 50, 796-805. [CrossRef] [PubMed]

23. Sharon-Asa, L.; Shalit, M.A.; Bar, E.; Holland, D.; Or, E.; Lavi, U. Citrus fruit flavor and aroma biosynthesis: Isolation, functional characterization, and developmental regulation of Cstps1, a key gene in the production of the sesquiterpene aroma compound valencene. Plant. J. 2003, 36, 664-674. [CrossRef] [PubMed]

24. Jing, L.; Lei, Z.; Zhang, G.; Pilon, A.C.; Huhman, D.V.; Xie, R.; Xi, W.; Zhou, Z.; Sumner, L.W. Metabolite profiles of essential oils in citrus peels and their taxonomic implications. Metabolomics 2015, 11, 952-963. [CrossRef]

25. Liu, C.; Jiang, D.; Cheng, Y.; Deng, X.; Chen, F.; Fang, L.; Ma, Z.; Xu, J. Chemotaxonomic study of Citrus, Poncirus and Fortunella genotypes based on peel oil volatile compounds-deciphering the genetic origin of Mangshanyegan (Citrus nobilis Lauriro). PLoS ONE 2013, 8, e58411. [CrossRef] [PubMed]

26. Gonzalez-Mas, M.C.; Rambla, J.L.; Alamar, M.C.; Gutierrez, A.; Granell, A. Comparative analysis of the volatile fraction of fruit juice from different Citrus species. PLoS ONE 2011, 6, e22016. [CrossRef] [PubMed]

27. González-Mas, M.C.; Rambla, J.L.; López-Gresa, M.P.; Blázquez, M.A.; Granell, A. Volatile Compounds in Citrus Essential Oils: A Comprehensive Review. Front. Plant Sci. 2019, 12. [CrossRef]

28. Shen, S.L.; Yin, X.R.; Zhang, B.; Xie, X.L.; Jiang, Q.; Grierson, D.; Chen, K.S. CitAP2.10 activation of the terpene synthase CsTPS1 is associated with the synthesis of (+)-valencene in 'Newhall' orange. J. Exp. Bot. 2016, 67, 4105-4115. [CrossRef]

29. Schnee, C.; Kollner, T.G.; Held, M.; Turlings, T.C.; Gershenzon, J.; Degenhardt, J. The products of a single maize sesquiterpene synthase form a volatile defense signal that attracts natural enemies of maize herbivores. Proc. Natl. Acad. Sci. USA 2006, 103, 1129-1134. [CrossRef]

30. Oliveros, J.C. An Interactive Tool for Comparing Lists with Venn Diagrams. Available online: http: //bioinfogp.cnb.csic.es/tools/venny/index.html (accessed on 12 December 2019).

31. Rohart, F.; Gautier, B.; Singh, A.; Lê Cao, K.-A. mixOmics: An R package for 'omics' feature selection and multiple data integration. PLoS Comput Biol. 2017, 13, e1005752. [CrossRef]

Sample Availability: Samples of the compounds not are available from the authors. 